Timo M. Breit - Ingrid L. M. Wolvers-Tettero

Ad J. J. C. Bogers - Ronald R. de Krijger

Juriy W. Wladimiroff - Jacques J. M. van Dongen

\title{
Rearrangements of the human TCRD-deleting elements
}

Received 25 January 1994 / Revised 18 February 1994

There are two types of T-cell receptors (TCR) present on human peripheral blood (PB) T lymphocytes: TCR- $\alpha \beta$ and TCR- $\gamma \delta$ (Davis and Bjorkman 1988). Although little is known about the mechanisms that commit a $\mathrm{T}$ cell to the $\alpha \beta$ - or $\gamma \delta$ lineage, it is generally assumed that the TCRD gene plays a pivotal role in the divergence of the two lineages (De Villartay et al. 1988; Hockett et al. 1988, 1989; Van Dongen et al. 1990). This assumption is based on two features of the TCRD gene. Firstly, the TCRD gene is located in the middle of the TCRA gene and is therefore deleted during $V \alpha-J \alpha$ rearrangement, which in principle excludes co-expression of TCRD and TCRA chains (Hockett et al. 1988; Isobe et al. 1988). Secondly, in normal polyclonal thymocytes a predominant rearrangement is observed, which represents the rearranged $\delta R E C$ and $\Psi J \alpha$ gene segments (De Villartay et al. 1987, 1988; Hockett et al. 1989). These two gene segments flank the major part of the TCRD gene and are called TCRD deleting elements, because their non-productive rearrangement deletes the intermediate germline and/or rearranged TCRD gene sequences (De Villartay et al. 1988). Therefore, a model is postulated in which a germline or a rearranged TCRD gene is deleted by the $\delta R E C-\Psi J \alpha$ rearrangement, which in turn can be replaced by a $V \alpha-J \alpha$ gene rearrangement (Fig. 1; Van Dongen et al. 1990).

T. M. Breit - I. L. M. Wolvers-Tettero - J. J. M. van Dongen (四) Department of Immunology, University Hospital Dijkzigt/Erasmus University, P. O. Box 1738, 3000 DR Rotterdam, The Netherlands

A. J. J. C. Bogers

Department of Thoracic Surgery, University Hospital Dijkzigt/Erasmus University, Rotterdam, The Netherlands

\section{R. R. de Krijger}

Department of Pediatrics, Sophia Children's Hospital/Erasmus

University, Rotterdam, The Netherlands

J. W. Wladimiroff

Department of Gynaecology/Obstetrics, Division of

Prenatal Diagnosis, University Hospital Dijkzigt/Erasmus University, Rotterdam, The Netherlands
Because new genetic nomenclature concerning $T C R$ gene segments has recently been introduced, we propose renaming the formerly designated $\delta R E C$ and $\Psi J \alpha$ gene segments according to this new nomenclature as defined by the WHO-IUIS Nomenclature Subcommittee on TCR Designation (WHO Bulletin 1993). The $\delta R E C$ gene segment could be renamed TCRDREC1, homologous to the $\delta R E C 1$ to $\delta R E C 3$ gene segments defined in the mouse (Takeshita et al. 1989). The $\Psi J \alpha$ gene segment could be renamed TCRAJ61P, as it is the most upstream-located gene segment of the 61 human $J \alpha$ gene segments (Koop et al. 1994). We designate the $\delta R E C-\Psi J \alpha$ rearrangement TCRDREC1AJ61P. Hereafter, we will use the WHO nomenclature for all TCR genes and rearrangements, with the abbreviations DRECl ( $\delta \mathrm{REC}), A J 61 P(\Psi \mathrm{J} \alpha)$, and DREC1.AJ61P ( $\delta \mathrm{REC}-\Psi \mathrm{J} \alpha)$.

Although the DREC1.AJ61P rearrangement by its result and prominent occurrence in the thymus seems to play a distinct role in the divergence of the human TCR- $\alpha \beta$ and TCR- $\gamma \delta$ T-cell lineages, limited information is available in the literature concerning this rearrangement. The first DREC1.AJ61P rearrangements were analyzed only by identification of circular excision products with a AJ61P.DREC1 signal joint (De Villartay et al. 1988). The subsequent determination of the DREC1.AJ61P junctional region of a T-cell line (DU.528) showed not only N-region nucleotide insertion and deletion of nucleotides by trimming of the flanking sequences comparable to a normal rearrangement, but revealed also evidence for TCRDD $(D \delta)$-gene-derived junctional region nucleotides (Begley et al. 1989). Other rearrangements involving one of the two $T C R D$ (TCR- $\delta$ )-deleting elements have also been observed,

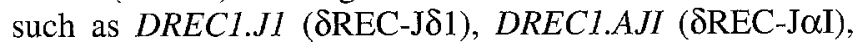
and DRECl.AJII ( $\delta$ REC-J $\alpha I I)$ in T-cell acute lymphoblastic leukemia (T-ALL; Breit et al. 1991 a, b; Hara et al. 1991),

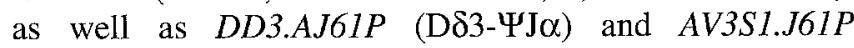
$(\mathrm{V} \alpha 3.1-\Psi \mathrm{J} \alpha)$ in normal thymocytes (De Villartay et al. 1988).

A total of 150 DREC1.AJ61P junctional regions were analyzed to determine their precise sequence and to investigate whether TCRDD gene segments can occur in 


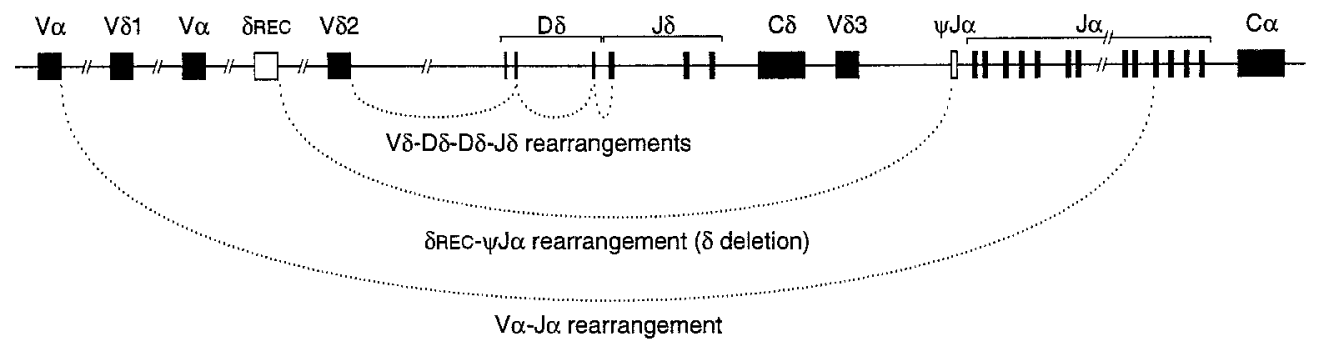

these junctional regions and if so, in what frequency they are present. To investigate at the same time potential differences in the DREC1.AJ61P rearrangement during human ontogeny, we studied 15 thymus and blood cell samples from fetuses, neonates, and adults.

The 15 cell samples consisted of five fetal thymi $(12,15$, 16,17 , and 18 weeks of gestation), five postnatal thymi ( 3 days, 1 month, 1 year, 5 years, and 15 years), one fetal cord blood sample (18 weeks of gestation), one neonatal cord blood sample, and three adult PB samples (16 years, 27 years, and 28 years). DNA was extracted from the obtained cell samples as described (Van Dongen and WolversTettero 1991). Fifteen $\mu \mathrm{g}$ of each DNA sample was digested with Eco RI, Hin dIII, and/or Bgl II and analyzed by Southern blot analysis, using the DRECI probe (TCRDRE) and AJ61P probe (TCRAPJ; Breit et al. 1993). Of each DNA sample, $0.25-0.5 \mu \mathrm{g}$ was amplified in a normal $100 \mu \mathrm{l}$ polymerase chain reaction (PCR) reaction mix, using the
Fig. 1 Schematic representation of the human TCRAD (TCR- $\alpha / \delta)$ locus. Indicated are the various gene segments including the TCRDdeleting elements: DRECI ( $\delta \mathrm{REC}$ ) and AJ61P ( $\Psi \mathrm{J} \alpha)$. The dotted lines indicate the possible consecutive gene rearrangements: TCRDV.D.D.J $(\mathrm{V} \delta \mathrm{D} \delta \delta-\mathrm{D} \delta-\mathrm{J} \delta), D R E C 1 . A J 61 P(\delta \mathrm{REC}-\Psi \mathrm{J} \alpha)$, and TCRAV.J $(\mathrm{V} \alpha-\mathrm{J} \alpha)$

Fig. $2 \mathrm{~A}$, B Southern blot analysis of the DRECI.AJ6IP rearrangement in various human cell samples. Lane 1, control DNA (cell line HELA); lane 2, fetal thymus 15 weeks; lane 3, fetal thymus 18 weeks; lane 4 , neonatal thymus 3 days; lane 5 , infant thymus 15 years; lane 6 , neonatal cord blood; lane 7, infant PB 16 years; lane 8, adult PB 28 years. A Hybridization of $H$ in dIII digests with the TCRDRE (DRECl) probe. B Rehybridization with the TCRAPJ (AJ6IP) probe. The band representing the preferential DRECI.AJ61P (SREC$\Psi \mathrm{J}(\alpha)$ rearrangement is indicated. All other rearranged bands represent other preferential rearrangements to either the DRECI or AJ6IP gene segment. ( $G$ indicates the germline band)

\section{A

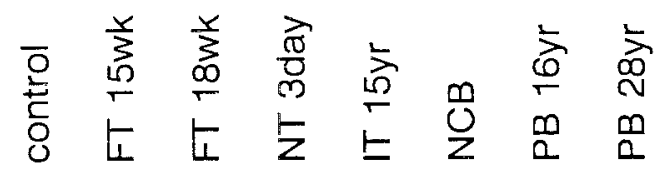

$-G$

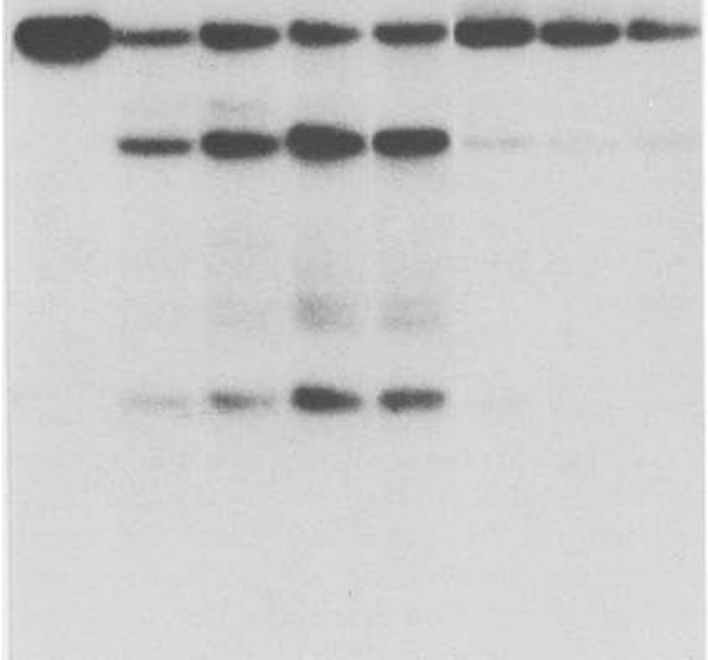

B
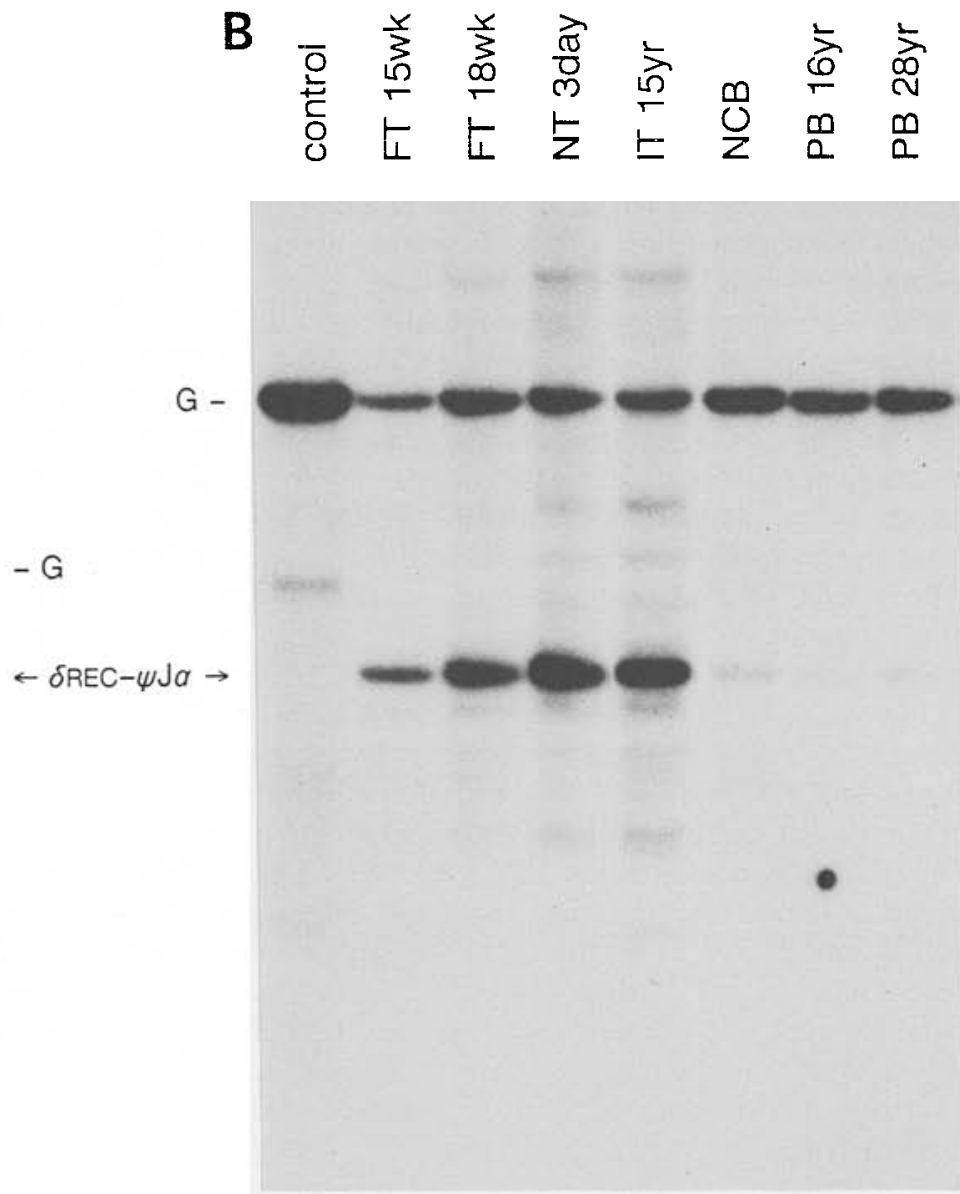
Table 1 Junctional region diversity of human $D R E C 1 . A J 61 P$ rearrangements, as determined by analyzing at least ten junctional regions per cell samples

\begin{tabular}{|c|c|c|c|c|c|c|c|}
\hline & \multicolumn{4}{|c|}{ No. of inserted nucleotides } & \multicolumn{3}{|c|}{ No. of deleted nucleotides } \\
\hline & $\mathrm{N}$-region & $T C R D D$ & P-region & Total & DRECl & AJ61P & Total \\
\hline \multicolumn{8}{|l|}{ Fetal thymus } \\
\hline 12 weeks & 1.3 & 0.3 & 0.1 & 1.7 & 4.8 & 4.7 & 9.5 \\
\hline 15 weeks & 1.7 & 0.8 & 0.2 & 2.7 & 1.8 & 4.3 & 6.1 \\
\hline 16 weeks & 1.6 & 0 & 0.4 & 2.0 & 1.9 & 2.9 & 4.8 \\
\hline 17 weeks & 1.7 & 1.7 & 0.3 & 3.7 & 3.7 & 2.1 & 4.8 \\
\hline 18 weeks & 2.3 & 1.0 & 0.3 & 3.6 & 2.0 & 2.4 & 4.4 \\
\hline \multicolumn{8}{|l|}{ Postnatal thymus } \\
\hline 3 days & 3.7 & 2.2 & 0.6 & 6.5 & 3.3 & 8.4 & 11.7 \\
\hline 1 month & 3.9 & 1.8 & 0.6 & 6.3 & 3.4 & 4.5 & 7.9 \\
\hline 1 year & 2.6 & 1.4 & 0.2 & 4.2 & 3.8 & 3.9 & 7.7 \\
\hline 5 years & 2.8 & 1.9 & 0.6 & 5.3 & 1.2 & 4.4 & 5.6 \\
\hline 15 years & 3.0 & 1.8 & 0 & 4.8 & 4.6 & 5.9 & 10.5 \\
\hline \multicolumn{8}{|l|}{ Fetal cord blood } \\
\hline 18 weeks & 2.5 & 1.7 & 0.4 & 4.6 & 3.7 & 5.1 & 8.8 \\
\hline \multicolumn{8}{|l|}{ Neonatal cord blood } \\
\hline NCB5 & 1.6 & 1.4 & 0.1 & 3.1 & 2.8 & 4.6 & 7.4 \\
\hline \multicolumn{8}{|l|}{ Peripheral blood } \\
\hline 16 years & 5.4 & 2.0 & 0.7 & 8.1 & 4.0 & 4.6 & 8.6 \\
\hline 27 years & 3.2 & 0.3 & 0.4 & 3.9 & 3.9 & 4.7 & 8.6 \\
\hline 28 years & 5.3 & 2.3 & 0.1 & 7.7 & 4.2 & 5.1 & 9.3 \\
\hline
\end{tabular}

oligonucleotide primers $\delta$ REC-5 $5^{\prime} \mathrm{E}$ (ctaagaatTCGATCCTCAAGGGTCGAGACTGTC) and $\Psi \mathrm{J} \alpha-3^{\prime} \mathrm{H}$ (cctgaagcTTAAGGCACATTAGAATCTCTCACTG) as described (Breit et al. 1993b). The obtained polyclonal PCR products of $\sim 500$ base pairs (bp) were digested with $E c o$ RI and Hin dIII and cloned in the pUC19 vector. Ten single bacteria colonies of each sample were randomly picked and sequenced with the universal pUC reverse sequencing primer as described (Sambrook et al. 1989).

Southern blot analysis of the various cell samples confirmed the prominent presence of the DRECI.AJ61P rearrangement in all thymic cell samples, but in PB mononuclear cells this rearrangement was hardly visible $(5 \%$ detection limit; Fig. 2). The latter observation is probably caused by the predominant biallelic TCRAV.J $(\mathrm{V} \alpha-\mathrm{J} \alpha)$ gene rearrangements in peripheral TCR- $\alpha \beta+$ T-lymphocytes, which have deleted the preexisting DREC1.AJ61P rearrangements.

The sequences of the DREC1.AJ61P junctional regions in the various cell samples are presented in Figure 3. Although there are some differences, in all cell samples $\mathrm{N}$ region and P-region nucleotide insertion occurred in addition to deletion of nucleotides from the flanking sequences. The characteristics of the DREC1.AJ61P junctional regions in Table 1 show that the total nucleotide insertion in fetal thymocytes $(1.7-3.7)$ is on average lower than in postnatal cell samples (4.2-6.5). Especially in 12 weeks thymocytes, $\mathrm{N}$-region nucleotide insertion was very low, probably due to low expression of the enzyme terminal deoxynucleotidyl transferase (Campana et al. 1989), which mediates the random $\mathrm{N}$-region nucleotide insertion. Nucleotide deletion was also more extensive in postnatal cell samples, suggesting that rearrangements in early fetal thymocytes are performed by an "immature" recombinase complex, which is less capable of nucleotide deletion. Furthermore, in almost all cell samples, nucleotide deletion by trimming of the DRECI gene segment was less extensive than trimming of the AJ61P gene segment, indicating that the activity of the recombinase enzyme complex has a direction, which may be related to the size of the spacers in the recombination signal sequences (Breit et al. 1993a).

Usually, TCRDD nucleotides are identified in TCRD junctional regions based on the guideline that at least onethird of the TCRDD gene segment has to be present with a minimum of three consecutive nucleotides. By using this guideline, we could identify putative $T C R D D$ gene-derived nucleotides in $36 \%$ of the DREC1.AJ61P junctional regions. However, there was no complete TCRDD gene segment present in any junctional region, whereas in normal TCRDV.J $(\mathrm{V} \delta$-J $\delta)$ junctional regions complete

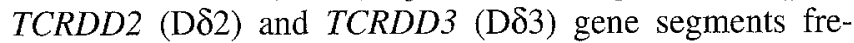
quently occur (Breit et al. $1991 \mathrm{~b}$; Panchamoorthy et al. 1991). Moreover, applying the same guideline to published TCRAV.J or even TCRGV.J $(\mathrm{V} \gamma \mathrm{J} \gamma)$ junctional regions, revealed comparable frequencies of putative TCRDD genederived nucleotides, although these junctional regions should not contain TCRDD gene segments (Breit et al. 1991 b; Porcelli et al. 1993). In fact, the DREC1.AJ61P junctional regions are highly homologous to TCRAV.J and TCRGV.J junctional regions (with only one $\mathrm{N}$-region) and are essentially smaller than TCRDV.J junctional regions (with primarily two or three $\mathrm{N}$-regions). We therefore conclude that most putative TCRDD gene-derived nucleotides observed in the DRECI.AJ61P junctional regions probably represent $\mathrm{N}$-region nucleotides, which is in line with the finding that putative TCRDD gene-derived nu- 

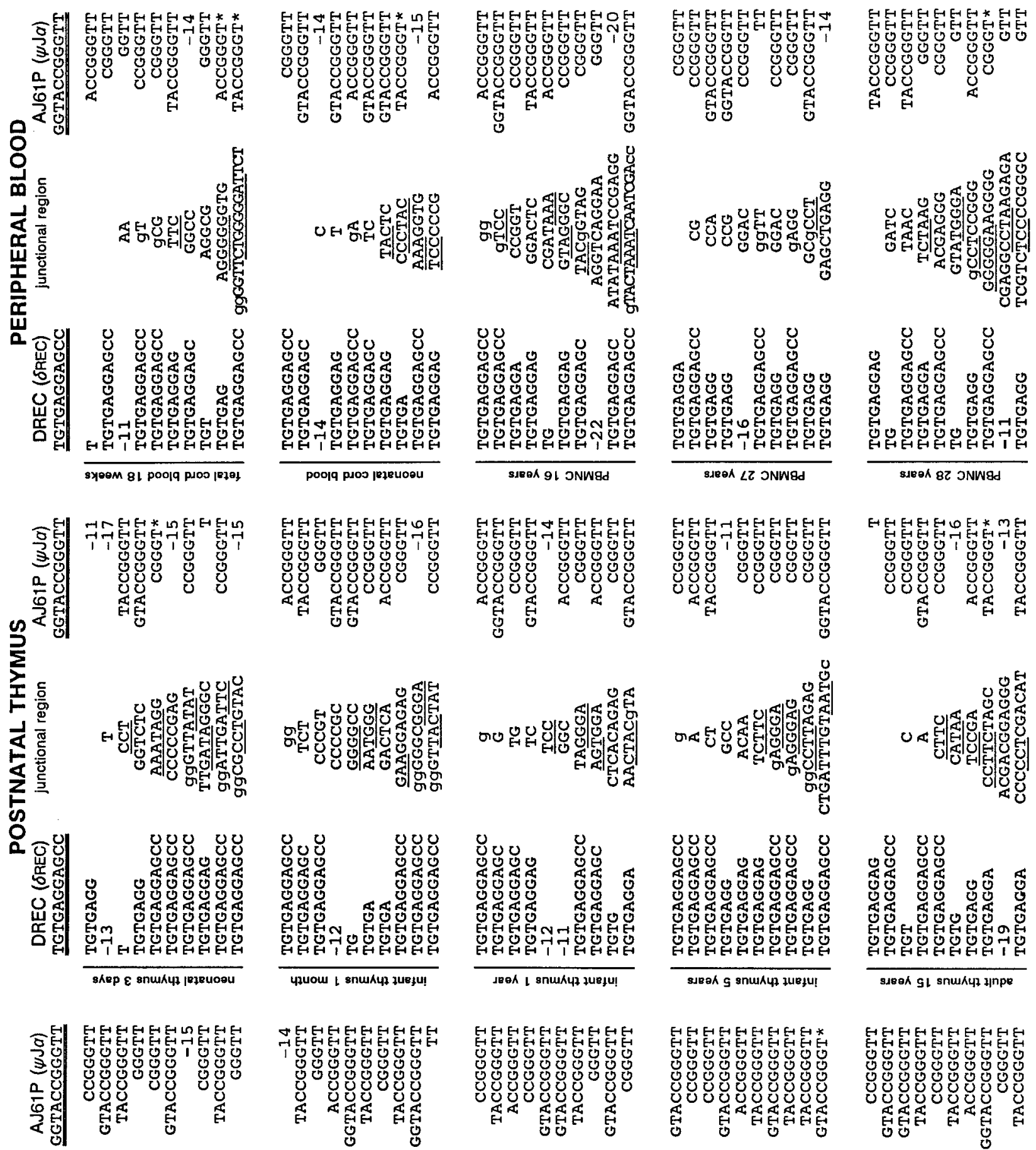

蛋
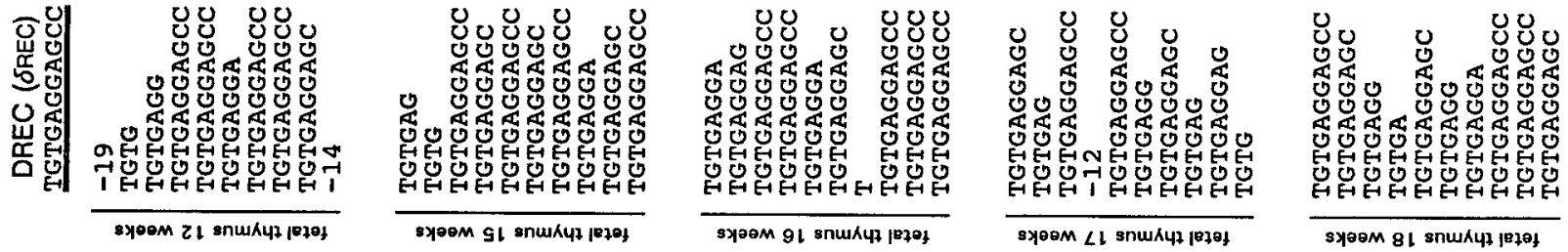
Fig. 3 Junctional region sequences of DRECI.AJ6IP rearrangements in various human cell samples. Sequences of the DRECI.AJ61P junctional regions are aligned with the known (double underlined) $D R E C 1$ ( $\delta \mathrm{REC}$ ) and AJ6IP ( $\Psi \mathrm{J} \alpha$ ) germline sequences. Single underlined sequences represent putative TCRDD gene-derived nucleotides. Lower-case characters represent P-region nucleotides and all other junctional region nucleotides represent $N$-region nucleotides. (* Indicates junctional regions $(\mathrm{n}=7)$ with a long string of $(\geq 5) T C R D D$ gene-derived nucleotides)

cleotides are virtually absent, if the number of N-region nucleotides is low (12-week-old fetal thymocytes).

Nevertheless, in some DREC1.AJ61P junctional regions (7/150) we discovered longer strings $(\geq 5)$ of putative $T C R D D$ gene nucleotides (Fig. 3), suggesting that in these particular instances TCRDD gene-derived nucleotides are indeed present. All three TCRDD gene segments were present at least once, indicating that the DRECl and AJ61P gene segments are able to rearrange to each TCRDD gene segment.

The finding that DRECI.AJ61P and TCRAVI junctional regions rarely include a $T C R D D$ gene segment may be due to the order of rearrangements on one allele: firstly a TCRDD.J (D $\delta$-J $\delta$ ) or TCRDV2/3.D.J (V $\delta 2 / 3-\mathrm{D} \delta$-J $\delta$ ) rearrangement, followed by the TCRD gene-deleting DREC1.AJ61P rearrangement, and finally a TCRAV.J rearrangement (Fig. 1). In principle, TCRDD gene segments can only be involved in DREC1.AJ61P or TCRAVJ rearrangements, if germline TCRDD gene segments are available at the time of rearrangement, i.e., germline TCRD genes or incompletely rearranged TCRD genes. Apparently this does not occur frequently, or there are other (yet unknown) restrictions excluding TCRDD gene segments from these rearrangements. Overall, the DREC1.AJ61P rearrangement appears to be just a TCRAV.J-like rearrangement, committing the thymocyte to the TCR- $\alpha \beta$ lineage.

It can be concluded that the predominant $T C R D$ genedeleting DRECl.AJ61P rearrangement is present in human thymocytes throughout ontogeny. The size of the DREC1.AJ61P junctional regions increases during thymic ontogeny, but no further ontogenic differences were observed. The TCRD gene-deleting elements can potentially rearrange to $T C R D D$ gene segments, but our extensive sequencing analyses of $150 D R E C 1 . A J 61 P$ junctional regions revealed that they rarely contain $T C R D D$ gene-derived nucleotides.

Acknowledgments The authors gratefully acknowledge Prof. Dr. R. Benner, Dr. H. Hooijkaas, and Ms. E. J. Mol for their continuous support; Prof. Dr. E. Bos for kindly providing the postnatal thymus samples; Ms. W. M. Comans-Bitter for collecting the fetal thymus samples; Mr. T. M. van Os for his excellent assistance in the preparation of the figures; and Ms. A. D. Korpershoek for her secretarial support.

\section{References}

Begley, C. G., Aplan, P. D., Davey, M. P., De Villartay, J.-P., Cohen, D. I., Waldmann, T. A., and Kirsch, I. R. Demonstration of $\delta$ RECpseudo $J \alpha$ rearrangement with deletion of the $\delta$ locus in a human stem-cell leukemia. J Exp Med 170: 339-342, 1989

Breit, T. M., Wolvers-Tettero, I. L. M., Hählen, K., Van Wering, E. R., and Van Dongen, J. J. M. Limited combinatorial repertoire of $\gamma \delta \mathrm{T}$ cell receptors expressed by T-cell acute lymphoblastic leukemias. Leukemia 5: 116-124, 1991 a

Breit, T. M., Wolvers-Tettero, I. L. M., Hählen, K., Van Wering, E. R., and Van Dongen, J. J. M. Extensive junctional diversity of $\gamma \delta$ T-cell receptors expressed by T-cell acute lymphoblastic leukemias: implications for the detection of minimal residual disease. Leukemia 5: $1076-1086,1991$ b

Breit, T. M., Mol, E. J., Wolvers-Tettero, I. L. M., Ludwig, W.-D., Van Wering, E. R., and Van Dongen, J. J. M. Site-specific deletions involving the tal-1 and sil genes are restricted to cells of the $\mathrm{T}$ cell receptor $\alpha / \beta$ lineage: $T$ cell receptor $\delta$ gene deletion mechanism affects multiple genes. $J$ Exp Med 177: 965-977, 1993 a

Breit, T. M., Wolvers-Tettero, I. L. M., Beishuizen, A., Verhoeven, M.A. J., Van Wering, E. R., and Van Dongen, J. J. M. Southern blot patterns, frequencies and junctional diversity of T-cell receptor $\delta$ gene rearrangements in acute lymphoblastic leukemia. Blood 82: $3063-3074,1993 b$

Campana, D., Janossy, G., Coustan-Smith, E., Amlot, P. L., Tian, W.T., Ip, S., and Wong, L. The expression of T cell receptor-associated proteins during $\mathrm{T}$ cell ontogeny in man. $J$ Immunol 142: 57-66, 1989

Davis, M. M. and Bjorkman, P. J. T-cell antigen receptor genes and Tcell recognition. Nature 334: 395-402, 1988

De Villartay, J.-P., Lewis, D., Hockett, R. D., Waldmann, T. A., Korsmeyer, S. J., and Cohen, D. I. Deletional rearrangement in the human T-cell receptor $\alpha$-chain locus. Proc Natl Acad Sci USA 84: $8608-8612,1987$

De Villartay, J.-P., Hockett, R. D., Coran, D., Korsmeyer, S. J., and Cohen, D. I. Deletion of the human T-cell receptor $\delta$-gene by a sitespecific recombination. Nature 335: 170-174, 1988

Hara, J., Takihara, Y., Yumura-Yagi, K., Ishihara, S., Tawa, A., Mak, T. W., Gelfand, E. W., Okada, S., and Kawa-Ha, K. Differential usage of $\delta$ recombining element and $\mathrm{V} \delta$ genes during T-cell ontogeny. Blood 78: 2075-2081, 1991

Hockett, R. D., De Villartay, J.-P., Pollock, K., Poplack, D. G., Cohen, D. I., and Korsmeyer, S. J. Human T-cell antigen receptor (TCR) $\delta$-chain locus and elements responsible for its deletion are within the TCR $\alpha$-chain locus. Proc Natl Acad Sci USA 85: 9694-9698, 1988

Hockett, R. D., Nuñez, G., and Korsmeyer, S. J. Evolutionary comparison of murine and human $\delta$ T-cell receptor deleting elements. The New Biologist I: 266-274, 1989

Isobe, M., Russo, G., Haluska, F. G., and Croce, C. M. Cloning of the gene encoding the $\delta$ subunit of the human T-cell receptor reveals its physical organization within the $\alpha$-subunit locus and its involvement in chromosome translocations in T-cell malignancy. Proc Natl Acad Sci USA 85: 3933-3937, 1988

Koop, B. F., Row, L., Wang, K., Kuo, C. L., Seto, D., Lenstra, J. A, Howard, S., Shan, W., Wilke, E., and Hood, L. The human T-cell receptor $\mathrm{C} \alpha / \mathrm{C} \delta$ region: organization, sequence and evolution of $97.6 \mathrm{~kb}$ of DNA. Genomics 19: 478-493, 1994

Panchamoorthy, G., McLean, J., Modlin, R. L., Morita, C. T., Ishikawa, S., Brenner, M. B., and Band, H. A predominance of the T cell receptor $\mathrm{V} \gamma 2 / \mathrm{V} \delta 2$ subset in human mycobacteria-responsive $\mathrm{T}$ cells suggests germline gene encoded recognition. J Immunol 147 : $3360-3369,1991$

Porcelli, S., Yockey, C. E., Brenner, M. B., and Balk, S. P. Analysis of T cell antigen receptor (TCR) expression by human peripheral blood CD4-8- $\alpha / \beta \mathrm{T}$ cells demonstrates preferential use of several V $\beta$ genes and an invariant TCR $\alpha$ chain. $J$ Exp Med 178: 1-16, 1993

Sambrook, J., Fritsch, E. F., and Maniatis, T. Molecular Cloning: A Laboratory Manual, 2nd edn. Cold Spring Harbor Laboratory, Cold Spring Harbor, 1989 
Takeshita, S., Toda, M., and Yamagishi, H. Excision products of the $\mathrm{T}$ cell receptor gene support a progressive rearrangement model of the $\alpha / \delta$ locus. EMBO $J$ 8: $3261-3270,1989$

Van Dongen, J. J. M., Comans-Bitter, W. M., Wolvers-Tettero, I. L. M., and Borst, J. Development of human T lymphocytes and their thymus-dependency. Thymus 16: 207-234, 1990
Van Dongen, J. J. M. and Wolvers-Tettero, I. L. M. Analysis of immunoglobulin and T cell receptor genes. Part I: Basic and technical aspects. Clin Chim Acta 198: 1-91, 1991

WHO-IUIS Nomenclature Subcommittee on TCR Designation. Nomenclature for T-cell receptor (TCR) gene segments of the immune system. Bulletin WHO 71: 113-115, 1993 